\title{
PRODUCTION DE JUVÉNILES DE VAIRON PHOXINUS PHOXINUS L. À PARTIR DE LARVES OBTENUES EN CONDITIONS CONTRÔLÉES.
}

\section{J.M. STALMANS (1) et P. KESTEMONT (2)}

(1) Chaire de zoologie générale et appliquée, Facultés des Sciences Agronomiques de Gembloux, B-5800 Gembloux, Belgique.

(2) Unité d'Ecologie des Eaux Douces, Facultés Universitaires, Notre-Dame de la Paix, 61 rue de Bruxelles, B-5000 Namur, Belgique

\section{RÉSUMÉ}

Le prégrossissement et la production de juvéniles de vairon européen Phoxinus phoxinus ont été réalisées à partir de larves produites par reproduction artificielle ou seminaturelle sur substrats de graviers. Les larves résorbées ont été soit déversées directement en étangs d'alevinage, soit prégrossies en bassins-bâches fertilisés selon différentes densités de mise en charge (de 15 à 200 larves $/ \mathrm{m}^{2}$ et de 160 à 1020 larves $/ \mathrm{m}^{2}$, respectivement pour les alevinages en étang et pour le prégrossissement en bassinsbâches). Indépendamment de la densité de mise en charge, la survie a varié de 48 à $72 \%$ après 4 à 6 mois d'alevinage en étang et de 78 à $92 \%$ au terme de 1 à 2 mois de prégrossissement. Un premier essai en auge d'élevage a mis en évidence la faible efficacité des aliments artificiels de commerce et la difficulté qu'ont les larves à ingérer du zooplancton de taille supérieure à $200 \mu \mathrm{m}$.

\section{PRODUCTION OF MINNOWS \\ PHOXINUS PHOXINUS L. FROM LARVAE OBTAINED UNDER CONTROLLED CONDITIONS.}

\section{SUMMARY}

The first feeding and the production of one summer European minnows Phoxinus phoxinus were carried out on basis of larvae obtained by artificial or semi-natural reproduction on gravel substrates. At the end of the yolk sac resorption, the larvae were stocked directly in nursing ponds or first-fed in fertilized plastic cover tanks at different stocking densities (from 15 to 200 larvae/m² and 160 to 1020 larvae/m², respectively for the stocking in ponds or tanks). Independently of the stocking density, the survival varied between 48 and $72 \%$ after 4 to 6 months of rearing in pond and between 78 and $92 \%$ after 1 to 2 months of first-feeding. A first trial in rearing tank showed a low effectiveness of commercial dry diets and the difficulty to rear minnow larvae with zooplankton larger than $200 \mu \mathrm{m}$.

\section{INTRODUCTION}

Le vairon européen, Phoxinus phoxinus L., est un petit cyprin de mœurs grégaires présent dans de nombreux ruisseaux et rivières de la zone à ombre et barbeau (zonation piscicole de HUET, 1949) ainsi que dans certains lacs et étangs. En toute saison, il est très largement utilisé par les pêcheurs sportifs comme poissons-appâts pour la capture, principalement de la truite, mais également de la perche, du sandre, du brochet et accessoirement de l'anguille. L'élevage et la commercialisation de cette espèce restent cependant marginaux malgré une demande nettement supérieure à l'offre. D'après LE LOUARN (com. pers.), le vairon constitue, avec le goujon, l'espèce pour laquelle l'écart entre la demande et l'offre est le plus largement positif. 
En dehors de son intérêt en tant qu'appât pour la pêche sportive, son élevage peut également être envisagé en tant que poisson de repeuplement. On observe en effet une régression généralisée de cette espèce partout en Europe. Les causes de cette régression sont multiples et peuvent être résumées comme suit :

- les aménagements et travaux hydrauliques des cours d'eau qui éliminent, réduisent ou envasent les frayères (gravières).

- la détérioration de la qualité de l'eau, suite aux pollutions aiguës ou diffuses.

- la surexploitation halieutique sous la forme de captures excessives, légales ou illégales en vue de sa commercialisation comme appât.

- les rempoissonnements surdensitaires en truites d'élevage, exerçant une prédation anormalement élevée sur des populations de vairons de plus en plus marginalisées (PHILIPPART et VRANKEN, 1983).

Jusqu'à présent, l'élevage du vairon n'a été pratiqué qu'à l'échelle extensive, ce poisson étant essentiellement produit en tant qu'espèce d'accompagnement dans les grands étangs à cyprins et ne représentant qu'une faible part de la production piscicole de l'étang. Les différentes raisons, d'ordre économique et écologique, énoncées précédemment ont motivé la réalisation de travaux de recherche visant le contrôle de la reproduction et de l'alevinage de cette espèce en conditions intensives ou semi-intensives.

Les résultats présentés ci-dessous constituent une synthèse de nombreux essais de productions d'alevins et de juvéniles de vairon (1 été) effectués à différents niveaux d'intensification, à savoir en étangs, en bassins-bâches fertilisés de manière à assurer une production élevée en zooplancton et en auges d'élevage avec apport d'une alimentation exogène dès la fin de la résorption de la vésicule vitelline.

\section{MATÉRIEL ET MÉTHODES}

\subsection{Origine des larves}

Les larves de Phoxinus phoxinus ont été produites par reproduction artificielle ou semi-naturelle à partir de plusieurs lots de géniteurs (âge $=2-3$ ans ; poids individuel = 3-10 g) maintenus en étangs ou en auges, ou capturés dans le milieu naturel (sur les frayères) en saison de reproduction.

\section{a) Reproduction artificielle}

La technique utilisée est similaire à celle décrite par WOYNAROVICH et HORVATH (1981) pour la carpe Cyprinus carpio. Elle consiste, chez la femelle, en une double injection intrapéritonéale à l'aide d'extraits pituitaires bruts de carpe à raison d'une dose préparatoire de 0,5 à $2,5 \mu \mathrm{g} / \mathrm{g}$ suivie après 2 heures par une dose définitive de 5 à $25 \mu \mathrm{g} / \mathrm{g}$ et d'une simple injection de 4 à $10 \mu \mathrm{g} / \mathrm{g}$ chez le mâle. La fécondation artificielle est effectuée par la méthode sèche puis les œufs sont incubés en carafe de Zoug ou sur claies horizontales à une température de 11 à $15^{\circ} \mathrm{C}$. La faible fécondité des femelles et les taux variables de fécondation (20 à $94 \%$ selon les essais) ont conduit au développement d'une méthode mieux adaptée à cette espèce de taille réduite et à son comportement de reproduction, à savoir la reproduction semi-naturelle sur substrat.

\section{b) Reproduction semi-naturelle sur substrat}

Les géniteurs matures (abdomen gonflé chez les femelles, parure nuptiale et émission de sperme chez les mâles) sont placés en auges d'élevage $\left(1 \mathrm{~m}^{2}\right.$, vol. utile $=$ 300 I.) à raison de 500 à 1.500 poissons $/ \mathrm{m}^{2}$. Des paniers de $0,12 \mathrm{~m}^{2}$ contenant plusieurs couches de graviers $(\varnothing 1-2 \mathrm{~cm})$ sont placés dans les auges et sont renouvelés quotidiennement dès l'apparition des premières pontes, de manière à reduire la prédation effectuée sur les œufs par les mâles. L'incubation des œufs est effectuée sur le substrat lui- 
même, déposé en incubateur horizontal (type californien, HUET, 1970), avec traitement quotidien des œufs avec une solution de vert malachite $\left(1 \mathrm{~g} / \mathrm{m}^{3}\right)$ en circuit ouvert. Avec une fécondité de 80 à 700 vésicules résorbées viables par femelle et par saison, une auge contenant 1.000 géniteurs de vairon (sex ratio mâle : femelle $=1: 2$ ) est à même de produire environ 250.000 larves en une saison de reproduction, soit en 5 à 6 pontes successives.

\subsection{Techniques d'alevinage}

La production de juvéniles de vairon a été effectuée en petits étangs d'alevinage (1 à 10 ares) à partir de différentes densités de mise en charge en larves à vésicules résorbées (15 à 200 larves $/ \mathrm{m}^{2}$ ). Des essais de prégrossissement des larves ont également été réalisés de manière à produire des alevins de 1 à 2 mois destinés à être déversés en étangs ou bassins de grossissement. Deux méthodes ont été utilisées : soit le déversement des larves à vésicules résorbées en bassins-bâches enrichis en zooplancton, soit l'apport d'aliment à des larves maintenues en conditions entièrement artificielles.

\section{a) Préparation des étangs d'alevinage}

La mise en eau est effectuée durant les mois de mai à juillet, 1 à 3 semaines avant le déversement des larves à vésicules résorbées. Une fertilisation organique est assurée par apport de fumier de cheval à raison de 50 à $100 \mathrm{~kg} / \mathrm{are}$. La récolte automnale des alevins est effectuée derrière le moine, à l'aide de filet poche à mailles de 1,5 à $3 \mathrm{~mm}$.

\section{b) Préparation des bassins-bâches}

Les bassins-bâches $\left(4 \times 2 \times 0,3 \mathrm{~m}\right.$, vol. utile $\left.=2 \mathrm{~m}^{3}\right)$ sont constitués de blocs de béton recouvert d'une bâche plastique imperméable. La mise en eau a lieu 3 semaines avant la mise en charge en larves à vésicule résorbée. Une augmentation de la production zooplanctonique est assurée par immersion de $4 \mathrm{~kg}$ de foin $/ \mathrm{m}^{3}$, favorisant le développement des protozoaires et des rotifères. Environ $90 \%$ du foin sont retirés lors de l'introduction des larves. Trois jours après la mise en charge, un traitement des bassins à raison de 0,5 ppm de Neguvon limite le développement du zooplancton de grande taille, ce qui favorise la multiplication des protozoaires et rotifères. Après 7 jours, une alimentation artificielle complémentaire (mélange de $50 \%$ de Mikromin-Tetra Werke et $50 \%$ d'un aliment de démarrage pour truite-Trouvit 000) est distribuée 2 à 3 fois/jour. Au terme du premier mois, un faible débit $(1-2 \mathrm{l} / \mathrm{min}$.) est maintenu. La température de l'eau est de $20^{\circ} \mathrm{C}$, avec des extrêmes de 17 à $24{ }^{\circ} \mathrm{C}$.

Quatre densités de mise en charge $\left(160 ; 280 ; 380\right.$ et 1.020 larves $\left./ \mathrm{m}^{2}\right)$ ont été utilisées, chaque densité étant répliquée.

\section{c) Premier essai en auges d'élevage}

Une expérience préliminaire en auges d'élevage $(2,15 \times 0,4 \times 0,17 \mathrm{~m}$; vol. utile $=$ 120 1.) a été réalisée à partir de 6 groupes de 800 larves à vésicule résorbée recevant chacun une alimentation vivante ou inerte :

- groupe 1 : nauplii d'Artemia salina (400 à $600 \mu \mathrm{m})$

- groupe 2 : zooplancton de 63 à $200 \mu \mathrm{m}$ (rotifères et nauplii de copépodes)

- groupe 3 : zooplancton de 200 à $400 \mu \mathrm{m}$ (jeunes copépodes et cladocères)

- groupe 4 : aliment artificiel (Tetramin, Tetra Werke) micronisé, particules de 50 à $200 \mu \mathrm{m}$.

- groupe 5 : aliment artificiel de démarrage pour truite (Trouvit starter, Trouw \& Co) micronisé, particules de 50 à $200 \mu \mathrm{m}$.

- groupe 6 : à jeun

Aucune réplication n'a pu être effectuée. 
Les nauplii d' $A$. salina sont obtenus par décapsulation puis incubation des cystes à $30{ }^{\circ} \mathrm{C}$, selon la technique décrite par SORGELOOS, 1980. Le plancton est produit en bassins-bâches et fractionné par filtration sur une succession de tamis $(\varnothing 400 \mathrm{~mm})$ à mailles décroissantes : $1 \mathrm{~mm}, 400 \mu \mathrm{m}, 200 \mu \mathrm{m}, 63 \mu \mathrm{m}$. Seules les fractions 63-200 $\mu \mathrm{m}$ et $200-400 \mu \mathrm{m}$ sont retenues. Les aliments vivants et inertes sont distribués 3 fois/jour, ad libidum.

\subsection{Evaluation des résultats}

Les résultats obtenus selon les différentes techniques d'alevinage ou de prégrossissement sont évalués en termes de taux de survie, de croissance spécifique et de production. Ces paramètres sont calculés comme suit :

Taux de survie $(\%)=100 \times \mathrm{N} t / \mathrm{Ni}$

Taux de croissance spécifique $(\% \mathrm{P} / \mathrm{j})=100 \times(\mathrm{LnPf}-\mathrm{Ln} \mathrm{Pi}) / \Delta \mathrm{t}$

Production $\left(\mathrm{g} / \mathrm{m}^{2} / \mathrm{j}\right)=(\mathrm{Bmf}-\mathrm{Bmi}) / \mathrm{S} . \Delta \mathrm{t}$

où $\mathrm{Ni}, f=$ nombre initial et final de poissons, $\mathrm{Pi}, f=$ poids moyen initial et final $(\mathrm{mg})$, $\mathrm{t}=$ temps (jours), Bmi, $\mathbf{f}=$ biomasse initiale et finale $(\mathrm{g}), \mathrm{S}=$ surface d'élevage $\left(\mathrm{m}^{2}\right)$. Un test de Student est utilisé pour comparer les résultats des alevinages en étang.

Les résultats de l'alevinage en bassins-bâches sont traités par une analyse de la variance à un critère de classification (DAGNELIE, 1975). Un test de Bartlett fut, au préalable, réalisé afin de vérifier l'homogénéité des variances.

\section{RÉSULTATS}

La compilation de l'ensemble des données récoltées dans le cadre des expériences de reproduction et de l'alevinage a permis d'établir une relation poids-longueur précise chez le vairon, depuis le stade de larve à vésicule résorbée $(P \mathrm{~m}=1,86 \mathrm{mg}, \mathrm{Lt}=7,2 \mathrm{~mm})$ jusqu'au poisson adulte $(\mathrm{Pm}=3,5 \mathrm{~g}$ et $\mathrm{Lt}=70 \mathrm{~mm})$. La taille commerciale est également mentionnée (fig. 1).

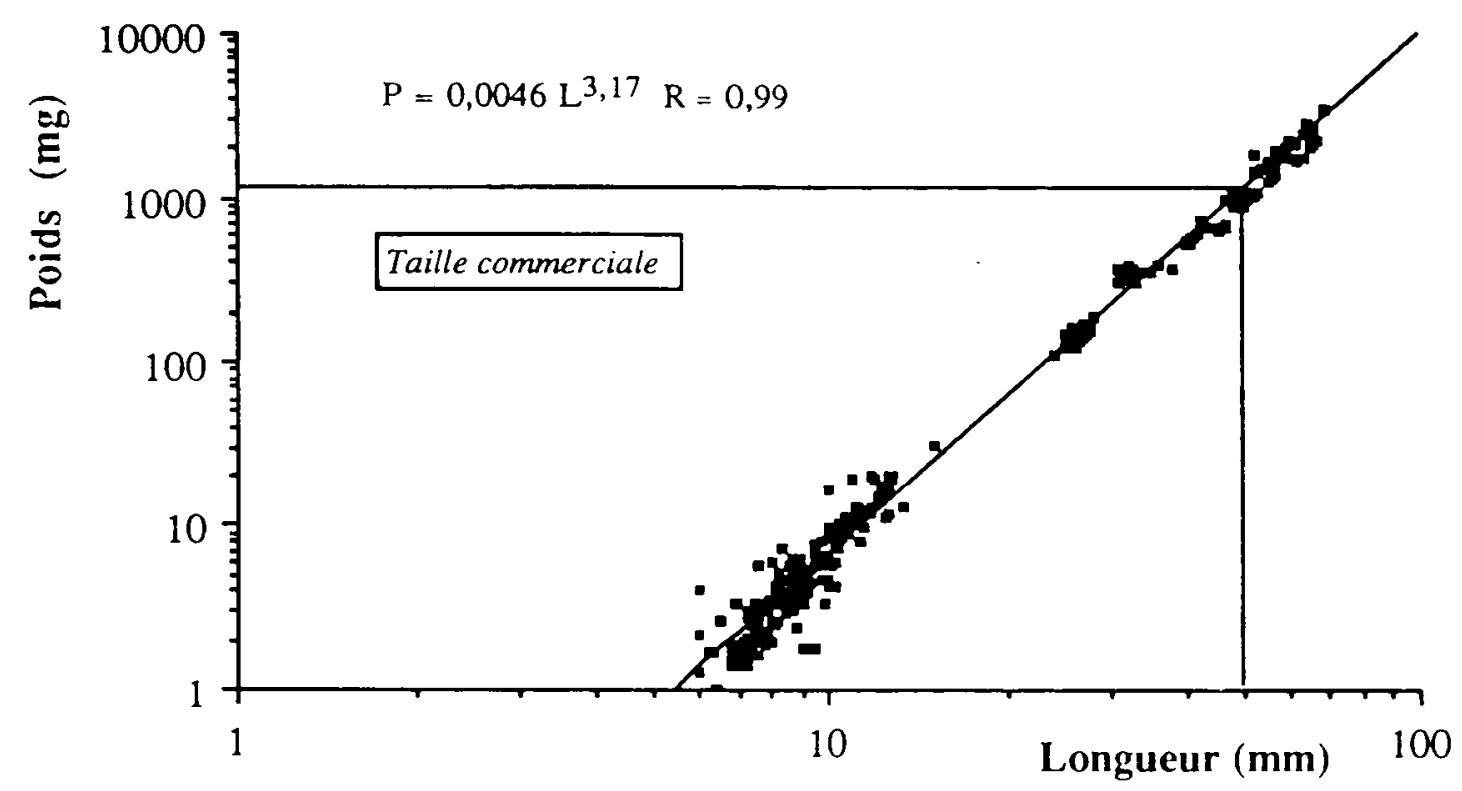

figure 1 : Relation poids-longueur du vairon Phoxinus phoxinus, depuis l'éclosion jusqu'au poisson adulte de quelques $\mathrm{g}$.

figure 1 : Weight-length relationship of the minnow Phoxinus phoxinus, from hatching to adult size (few $\mathrm{g}$ ). 


\subsection{Alevinage en étangs}

Le tableau I présente les résultats de survie, croissance et production de juvéniles de vairons (1 été) à partir de différentes densités de mise en charge en larves à vésicule résorbée. La survie semble indépendante de la densité de mise en charge et varie de 45,5 à $71,6 \%$. De façon similaire, le taux de croissance spécifique $(\mathrm{g})$ est relativement stable quelle que soit la densité initiale. II faut toutefois remarquer que, dans les essais effectués à faible densité (15 poissons $/ \mathrm{m}^{2}$ ), la récolte a été effectuée plus tardivement que dans les autres groupes (novembre-décembre au lieu de septembre-octobre), alors que la température de l'eau a généralement été inférieure à $10-12{ }^{\circ} \mathrm{C}$ dès le mois d'octobre, limitant de la sorte la croissance des poissons en novembre et décembre. La figure 2 indique une nette augmentation de la production à mesure que la densité de mise en charge augmente.

Tableau I : Influence de la densité de mise en charge en larves à vésicule résorbée sur la survie, la croissance et la production de juvéniles (1 été) de vairon Phoxinus phoxinus L. lors d'un alevinage direct en étang.

Table I : Influence of stocking density of larvae on survival, growth and production of one summer minnows Phoxinus phoxinus L. directly stocked in pond since yolk sac resorption.

\begin{tabular}{ccccccccc}
\hline $\begin{array}{c}\text { Densité } \\
\left(\mathrm{n} / \mathrm{m}^{2}\right)\end{array}$ & $\begin{array}{c}\text { Essais } \\
(\mathrm{n})\end{array}$ & $\begin{array}{c}\text { Pmi } \\
(\mathrm{mg})\end{array}$ & $\begin{array}{c}\Delta \mathrm{t} \\
(\mathrm{j})\end{array}$ & $\begin{array}{c}\text { Survie } \\
(\%)\end{array}$ & $\begin{array}{c}\text { Pmf } \\
(\mathrm{g})\end{array}$ & $\begin{array}{c}\text { Biomasse } \\
\text { finale }(\mathrm{kg} / \mathrm{j})\end{array}$ & $\begin{array}{c}\text { Croissance } \\
(\% \mathrm{P} / \mathrm{j})\end{array}$ & $\begin{array}{c}\text { Production } \\
\left(\mathrm{g} / \mathrm{m}^{2} / \mathrm{j}\right)\end{array}$ \\
\hline 15 & 4 & 1,86 & 180 & $48,1^{\mathrm{a}}$ & 0,52 & 37,5 & $3,1^{\mathrm{a}}$ & $0,021^{\mathrm{a}}$ \\
40 & 4 & 1,86 & 140 & $58,9^{\mathrm{a}}$ & 0,83 & 195,5 & $4,4^{\mathrm{b}}$ & $0,140^{\mathrm{b}}$ \\
100 & 5 & 1,86 & 130 & $65,6^{\mathrm{a}}$ & 0,30 & 196,8 & $3,7^{\mathrm{a}}$ & $0,134^{\mathrm{b}}$ \\
150 & 7 & 1,86 & 115 & $45,5^{\mathrm{a}}$ & 0,49 & 334,5 & $4,8^{\mathrm{b}}$ & $0,287^{\mathrm{c}}$ \\
200 & 1 & 1,86 & 130 & 71,6 & 0,34 & 486,9 & 4,0 & 0,372 \\
\hline
\end{tabular}

Les moyennes portant les mêmes lettres ne sont pas significativement différentes $(P>0,05)$

Pmi, $f=$ poids moyen initial et final

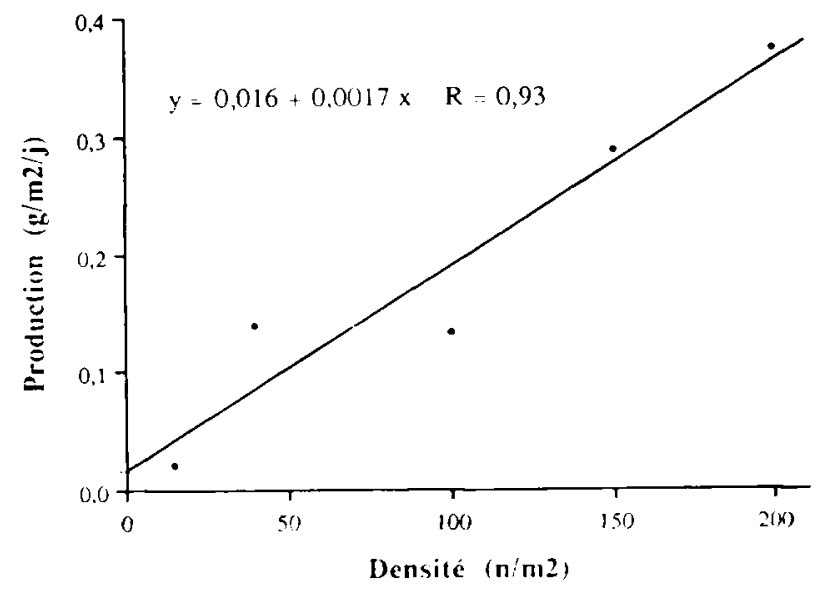

figure 2 : Influence de la densité de mise en charge $\left(n / \mathrm{m}^{2}\right)$ en larves à vésicule résorbée sur la production ( $\left.\mathrm{g} / \mathrm{m}^{2} / \mathrm{jour}\right) \mathrm{du}$ vairon Phoxinus phoxinus lors d'un alevinage direct en étang.

figure 2 : Influence of stoking density $\left(\mathrm{n} / \mathrm{m}^{2}\right)$ of larvae on production $\left(\mathrm{g} / \mathrm{m}^{2} /\right.$ day $)$ of minnow Phoxinus phoxinus directly stocked in pond since yolk sac resorption. 


\subsection{Prégrossissement en bassins-bâches}

Le prégrossissement en bassins-bâches fertilisés par immersion de foin avant le déversement des larves et nourrissage complémentaire après environ 1 semaine procure des taux de survie satisfaisants, variant de 78,3 à $92,1 \%$ après 30 à 64 jours d'alevinage (tableau II). A hautes densités (1020 larves $/ \mathrm{m}^{2}$ ), la croissance est toutefois relativement faible, rendant la récolte des alevins particulièrement délicate. A cette densité, la biomasse de zooplancton a diminué très rapidement et la croissance des larves était entièrement dépendante de la qualité et de la quantité d'aliment distribué. La meilleure croissance et production a été obtenue à la densité de 285 larves $/ \mathrm{m}^{2}$, avec un taux de croissance spécifique de $8,5 \% /$ jour (tableau II). L'analyse de variance révèle un effet hautement significatif $(P<0,01)$ de la densité de mise en charge sur la production en bassins-bâches.

Tableau II : Prégrossissement de larves de vairon Phoxinus phoxinus L. en bassinsbâches $\left(8 \mathrm{~m}^{2}-2 \mathrm{~m}^{3}\right)$ selon différentes densités de mise en charge.

Table II : First-feeding of Phoxinus phoxinus larvae in plastic cover tank $\left(8 \mathrm{~m}^{2-}\right.$ $2 \mathrm{~m}^{3}$ ) at different stocking densities.

\begin{tabular}{|c|c|c|c|c|c|c|c|c|}
\hline \multirow[t]{2}{*}{ Exp. } & \multirow{2}{*}{$\begin{array}{c}\text { Essais } \\
(n)\end{array}$} & \multicolumn{2}{|c|}{ Mise en charge } & & \multirow{2}{*}{$\begin{array}{c}\Delta t \\
(j) \\
\text { Survie } \\
(\%)\end{array}$} & \multicolumn{2}{|c|}{ Récolte } & \multirow{2}{*}{$\begin{array}{l}\text { Production } \\
\left(\mathrm{g} / \mathrm{m}^{2} / \mathrm{j}\right)\end{array}$} \\
\hline & & $\begin{array}{l}\text { Densité } \\
\left(n / m^{2}\right)\end{array}$ & $\begin{array}{l}\text { Pmi } \\
(\mathrm{mg})\end{array}$ & & & $\begin{array}{l}\mathrm{Pmf} \\
(\mathrm{mg})\end{array}$ & $\begin{array}{c}\text { Croissance } \\
(\% \mathrm{P} / \mathrm{j})\end{array}$ & \\
\hline 1 & 2 & 160 & 1,86 & 64 & $78,3^{a}$ & $265^{a}$ & $7,7^{a}$ & $0,51^{\mathrm{a}}$ \\
\hline 2 & 2 & 280 & 1,86 & 52 & $82,6^{a b}$ & $155^{b}$ & $8,5^{a}$ & $0,69^{b}$ \\
\hline 3 & 2 & 380 & 1,86 & 54 & $92,1^{b}$ & $80^{c}$ & $6,9^{a}$ & $0,49^{a}$ \\
\hline 4 & 2 & 1020 & 1,86 & 30 & $90,9^{b}$ & $13,5^{d}$ & $6,5^{a}$ & $0,35^{c}$ \\
\hline
\end{tabular}

Les moyennes portant les mêmes lettres ne sont pas significativement différentes $(P>0,05)$ Pmi, $f=$ poids moyen initial et final

\subsection{Essais préliminaires en auges d'alevinage}

Les premiers essais d'alimentation en auges d'alevinage mettent en évidence la difficulté qu'ont les larves de vairon à s'alimenter à partir de zooplancton de taille supérieure à $200 \mu \mathrm{m}$ comme première source de nourriture exogène (tableau III). Les taux de survie et de croissance obtenus avec du zooplancton de 200 à $400 \mu \mathrm{m}$ ou des nauplii d'A. salina (400 à $500 \mu \mathrm{m})$ indiquent que seule une fraction de la population est apte à attraper et ingérer des proies de cette taille. Dans les groupes recevant une alimentation artificielle, la croissance est également très faible, les larves n'atteignent que 3,3 à $3,8 \mathrm{mg}$ après 11 à 17 jours de nourrissage, et la survie est inférieure à celle des larves maintenues à jeun. Les restes d'aliment ont par contre favorisé la multiplication des bactéries, ce qui peut avoir jouer un rôle défavorable sur la survie des larves. La nourriture non consommée se déposant au fond de l'auge a probablement empêché le développement d'algues comme ce fut observé dans les groupes à jeun où les larves avaient tendance à brouter les algues se développant sur le fond des bassins. Similairement à ce qui est observé chez les larves nourries avec du plancton de grande taille, les variations de croissance sont très importantes. 
Tableau III : Influence de l'aliment (vivant ou inerte) sur la survie et la croissance des larves de vairon Phoxinus phoxinus en auges d'élevage.

Table III : Influence of live and dry food on survival and growth of Phoxinus phoxinus larvae reared in artificial tanks.

\begin{tabular}{lccccccc}
\hline Aliment & $\begin{array}{c}\Delta t \\
(\mathrm{j})\end{array}$ & $\begin{array}{c}\text { Survie } \\
(\%)\end{array}$ & $\begin{array}{c}\text { Pmi } \\
(\mathrm{mg})\end{array}$ & $\begin{array}{c}\text { Lti } \\
(\mathrm{mm})\end{array}$ & $\begin{array}{c}\text { Pmf } \\
(\mathrm{mg})\end{array}$ & $\begin{array}{c}\text { Ltf } \\
(\mathrm{mm})\end{array}$ & $\begin{array}{c}\text { Croissance } \\
(\% \mathrm{P} / \mathrm{j})\end{array}$ \\
\hline $\begin{array}{l}\text { Zooplancton } \\
(63-200 \mu \mathrm{m})\end{array}$ & 17 & 78 & 1,86 & 7,2 & 9,6 & 10,9 & 9,5 \\
$\begin{array}{l}\text { Zooplancton } \\
(200-400 \mu \mathrm{m})\end{array}$ & 17 & 44 & 1,86 & 7,2 & 12,8 & 11,8 & 11,2 \\
$\begin{array}{l}\text { A. salina } \\
(400-600 \mu \mathrm{m})\end{array}$ & 15 & 30 & 1,86 & 7,2 & 13,5 & 12,0 & 13,2 \\
$\begin{array}{l}\text { Trouvit starter micronisé } \\
\text { Tetramin micronisé }\end{array}$ & 17 & 34 & 1,86 & 7,2 & 3,8 & 8,4 & 4,1 \\
Jeûne & 11 & 60 & 1,86 & 7,2 & 3,3 & 8,1 & 5,2 \\
\hline
\end{tabular}

Pmi, $f=$ poids moyen initial et final

Lti, $f=$ longueur totale initiale et finale

\section{DISCUSSION}

D'après les résultats obtenus lors des nombreux essais effectués avec des larves à vésicule résorbée de vairon Phoxinus phoxinus, il apparaît que l'alevinage direct en étang fertilisé est une technique simple et procurant des taux de survie et de production satisfaisants, d'autant plus que cette espèce présente une croissance très lente si on la compare à d'autres larves de cyprinidés telles que la carpe Cyprinus carpio, l'orfe Idus idus ou le gardon Rutilus rutilus (TIMMERMANS, 1989). La période comprise entre la résorption de la vésicule vitelline et le moment où l'alevin a atteint un poids de quelques centaines de mg est donc nettement plus longue chez le vairon que chez ces autres espèces. Or, il a été montré que, durant cette période, les larves de cyprins sont très sensibles aux conditions extérieures telles que l'absence de nourriture adéquate, la prédation (y compris celle exercée par les insectes aquatiques et certains copépodes) et les accidents climatiques (HORVATH, 1977 ; GILLET, 1980 ; TORRANS, 1986). Des conditions défavorables (chute brutale de température, agitation de l'eau) peuvent en effet anéantir jusqu'à $90 \%$ de la population d'un étang d'alevinage de carpes, lorsque les larves sont déversées en étang à la fin de la période de résorption (HORVATH, 1977). A ce titre, les larves de Phoxinus phoxinus présentent une résistance quasi exceptionnelle. Des déversements en larves à vésicule résorbée ont parfois été suivis directement par des périodes de gel intense sans que la population déversée en soit fortement affectée. La taille relativement élevée des larves de vairon $(P m=1,9 \mathrm{mg}$ ) en comparaison à celle d'autres cyprins (carpe $=1,0 \mathrm{mg}$, carassin doré $=1,2 \mathrm{mg}$, goujon $=0,5 \mathrm{mg}$ ) et leur aptitude à vivre dans des zones relativement fraîches (zone à ombre) peuvent probablement expliquer cette résistance élevée aux mauvaises conditions. Malgré la petite taille des adultes et leur faible longévité, la stratégie de reproduction du vairon serait à rapprocher d'une stratégie $\mathrm{K}$ (fécondité faible, progéniture de grande taille, MAC ARTHUR et WILSON, 1967) plutôt que de la stratégie $r$ caractérisant généralement la reproduction des cyprins. 
Compte tenu de la faible capacité des larves de vairon à ingérer du zooplancton d'une taille supérieure à $200 \mu \mathrm{m}$ dès le démarrage (tableau 3), il pourrait s'avérer intéressant de préparer les étangs de premier alevinage par traitement préalable à l'aide d'insecticide (Flibol E, Neguvon, trichlorophon) ou par chaulage $(\mathrm{CaOH})$ de manière à éliminer les populations de crustacés et insectes et favoriser le développement des rotifères (TAMAS et HORVATH, 1976 ; KRAZHAN et ISAYEVA, 1978 ; BALVAY, 1980) comme ce fut pratiqué dans les bassins-bâches. Cette méthode permet en effet d'obtenir en 4 semaines d'élevage une amélioration de 30 à $50 \%$ des taux de survie des alevins pour des charges de l'ordre de $5.10^{6}$ larves/ha (soit 500 larves/m²) (GILLET, 1980).

Bien qu'il soit généralement admis que, chez les poissons non prédateurs, des mortalités importantes puissent survenir, essentiellement au stade d'alevin, suite à une situation de surpeuplement (BACKIEL et LE CREN, 1978), la survie des vairons n'a pas été réduite lors d'une augmentation de la densité de mise en charge. Notons cependant que la valeur maximale expérimentée est de 200 larves $/ \mathrm{m}^{2}$, ce qui représente une densité inférieure à celle préconisée pour les larves de carpe (400 à $600 / \mathrm{m}^{2}$, WOYNAROVICH et HORVATH, 1981). En ne considérant que la période comprise entre la mise en charge printanière et la récolte automnale, la production annuelle varie de $76,6 \mathrm{~kg} / \mathrm{ha}$ (densité initiale de 15 larves $/ \mathrm{m}^{2}$ ) à $1.360 \mathrm{~kg} / \mathrm{ha}$ (densité de 200 larves $/ \mathrm{m}^{2}$ ). En Europe septentrionale, la croissance n'est toutefois effective que durant 6 à 7 mois par an (avril à octobre), ce qui permet toutefois d'atteindre une production d'environ $800 \mathrm{~kg} / \mathrm{ha} / \mathrm{an}$ lors d'une mise en charge à hautes densités et si un nourrissage complémentaire est assuré régulièrement.

Le prégrossissement en bassins-bâches procure des résultats intéressants tant au niveau de la survie que de la croissance puisque les vairons peuvent atteindre un poids de 150 à $250 \mathrm{mg}$ en deux mois d'élevage. Bien que des essais de déversement en étang d'alevins prégrossis n'aient pas encore été réalisés, il est fort probable que la survie des alevins prégrossis soit nettement supérieure à celle observée lors du déversement direct des vairons au stade de larves à vésicule résorbée.

L'expérience préliminaire en auge d'alevinage avec apport de différentes fractions de plancton confirme l'efficacité d'un traitement préalable des bassins-bâches avec un insecticide sélectif qui tue aussi les crustacés, les helminthes, les hérudinae. L'importance d'une compatibilité entre la taille des proies et l'ouverture buccale des larves a déjà été rapportée par HARTMAN (1983) et DABROWSKI et BARDEGA (1984). Des expériences récentes (KESTEMONT et STALMANS, in prep.) ont toutefois montré que les larves de vairon étaient capables de consommer des nauplii d'A. salina lorsque ceux-ci étaient distribués sous forme congelée. Des taux de survie et de croissance très satisfaisants (96\% et $30 \mathrm{mg}$ après 28 jours à une densité de 50 larves/l) ont en effet été obtenus avec une alimentation mixte constituée de nauplii d' $A$. salina congelés et d'aliment artificiel spécialement conçu pour les larves de petite taille. La nécessité d'utiliser des aliments spécialement conçus pour les larves de cyprinidés (granulométrie très fine, homogénéité des particules, bonne stabilité dans l'eau) ainsi que des dispositifs d'élevage adaptés à leurs exigences (fréquence très élevée de nourrissage, conditions zootechniques optimales) a déjà été mis en évidence par plusieurs auteurs (DABROWSKI, 1984 ; CHARLON et BERGOT, 1986 ; CHARLON et al. 1986). La possibilité d'élever les larves de vairon en conditions artificielles présente un intérêt indéniable pour la production de cette espèce. DUMORTIER et al. (1991) ont en effet montré qu'il était possible d'obtenir des reproductions massives de vairon en période hivernale, par contrôle photothermique de la maturité des géniteurs. Un prégrossissement des larves en conditions thermorégulées durant 1 à 2 mois permettrait ainsi d'effectuer des mises en charge en alevins de 30 à $200 \mathrm{mg}$ très tôt dans la saison (dès le mois de mars) et d'obtenir des poissons de taille commerciale en une seule saison de croissance.

\section{REMERCIEMENTS}

Les auteurs remercient le Prof. Ch. GASPARD (Fac. Sc. Agr. Gembloux) ainsi que le personnel du Service de la Pêche pour l'aide et les conseils apportés à la réalisation de ce travail. Le financement de cette étude a été supporté par le Ministère de la Région Wallonne de Belgique. 


\section{BIBLIOGRAPHIE}

BACKIEL T. et LE CREN E.D., 1978. Some density relationship for fish populations parameters. In Ecology of freshwater fish production, Gerking Ed., Blackwell Sci. Publ., Oxford, 279-302.

BALVAY G., 1980. Fonctionnement et contrôle du réseau trophique en étang. In R. Billard, La pisciculture en étang, INRA Publ. Paris, 47-79.

CHARLON N. et BERGOT P., 1986. An improved automatic dry food dispenser for fish larvae. Progr. Fish Cult., 48, 156-158.

CHARLON N., DURANTE H., ESCAFFRE A.M., BERGOT P., 1986. Alimentation artificielle des larves de carpe ( Cyprinus carpio L.). Aquaculture, 54, 83-88.

DABROWSKI K., 1984. The feeding of fish larvae : present "state of the art" and perspectives. Reprod. Nutr. Develop., 24 (6), 807-833.

DABROWSKI K, et BARDEGA R., 1984. Mouth size and recommendation of feed size preferences in three cyprinid fish. Aquaculture, 40, 27-40.

DAGNELIE R., 1975. Théorie et méthodes statistiques. Presses Agronomiques de Gembloux, 2, $463 \mathrm{p}$.

DUMORTIER D., KESTEMONT P. et STALMANS J.M., 1991. Repeated off-season spawnings in the European minnow, Phoxinus phoxinus held in captivity (soumis).

GILLET C., 1980. Ecloserie et production de larves. In R. Billard, La Pisciculture en étang, INRA Publ., Paris, 175-188.

HARTMAN J., 1983. Two feeding strategies of young fishes. Arch. Hydrobiol., 96, 496-509.

HORVATH L., 1977. Propagation and reproduction biology of our breeded fish species in Hungarian experiences of fresh water fish breeding. I.C.A. Budapest, Hungary.

HUET M., 1949. Aperçu des relations entre la pente et les populations piscicoles des eaux courantes. Schweiz Z. Hydrol., 11, 3-4, 332-351.

HUET M., 1970. Traité de pisciculture. Bruxelles, Ch. de Wingaert, $718 \mathrm{p}$.

KRAZHAN S.A. et ISAYEVA S.A., 1978. The effects of the liming of fish-breeding ponds on some crustacean plankters. Hydrobiol. J. 14 (1), 31-33.

MAC ARTHUR R.H. et WILSON E.O., 1967. Theory of island biogeography. Princeton University Press, Princeton, $325 \mathrm{p}$.

PHILIPPART J.C. et VRANKEN M., 1983. Atlas des poissons de Wallonie. Distribution, Ecologie, Ethologie, Pêche, Conservation. Cah. Ethol. Appl., 3, 1-2, 3959.

SORGELOOS P., 1980. The use of the brine shrimp Artemia, in aquaculture. In Persoone G., Sorgeloos P., Roels E. and Jaspers E. The brine shrimp Artemia vol. 3, Ecology, culturing, Use in Aquaculture, Univers. Press, Wetteren, Belgium, 25-46.

TAMAS G. et HORVATH L., 1976. Growth of cyprinids under optimal zooplancton conditions Bamidgeh, 28, 50-56.

TIMMERMANS J.A., 1989. Données sur la croissance de quelques espèces de poissons dans des étangs de Campine. Min. Agr., Stion Rech. Forest. Hydrobiol., Sér. D., 56, $34 \mathrm{p}$.

TORRANS E.L., 1986. Fish/Plankton Interactions. in J. Lannans, R.O. Smitherman, G. Tchobanoglous, Principles and practices of pond aquaculture, 67-81.

WOYNAROVICH E. et HORVATH L., 1981. La reproduction artificielle des poissons d'eau chaude : manuel de vulgarisation. FAO Doc. Tech., Pêches, 201, 191 p. 